\title{
Hacia un mapa de intervenciones electorales locales en materia de seguridad urbana (Ciudad de Buenos Aires, 2007)
}

\author{
Mercedes Calzado \\ Instituto de Investigaciones Gino Germani. Consejo Nacional de Investigaciones \\ Científicas y Técnicas. Universidad de Buenos Aires. Buenos Aires, Argentina. \\ Email: mcalzado@sociales.uba.ar

\section{Juan Pedro Gallardo} \\ Instituto de Investigaciones Gino Germani. Universidad de Buenos Aires. \\ Buenos Aires, Argentina. \\ Email: gallardojp@hotmail.com
}

\begin{abstract}
Resumen: Durante la década del noventa de manera similar a lo sucedido en la región latinoamericana, las agendas políticas, mediáticas y sociales de la Ciudad de Buenos Aires pasaron a estar teñidas de la problemática de la seguridad urbana. Estas tensiones cobraron cuerpo en 2007 durante las elecciones a jefe de Gobierno de la Ciudad, momento en que se abrió una nueva etapa en la política local y nacional gracias al primer triunfo de Propuesta Republicana (PRO). Durante esta elección, la violencia urbana y los modos de intervención frente a esta problemática fueron medulares. Con diferencias y similitudes, los espacios políticos incorporaron esta dimensión temática a sus estrategias electorales. Desde un relevamiento de dispositivos gráficos, audiovisuales y digitales de cada espacio político, se reflexiona acerca de las modalidades enunciativas y temáticas en que se despliega el debate de la seguridad urbana como centro de la política local contemporánea.
\end{abstract}

Palabras clave: Inseguridad. Política, comunicación, campañas, ciudad de Buenos Aires.

\section{Towards a map of local electoral interventions on urban safety (City of Buenos Aires. 2007)}

\begin{abstract}
During the nineties, in similarity to what happened in Latin America, the insecurity topic appeared within political, media and social agendas in the City of Buenos Aires. The elections for head of government of the City in 2007 were relevant to think about this problem, moreover a new political era opened in local and national politics through the first triumph of Republican Proposal (PRO). During this election, urban violence and ways of intervention against this problem were in the core. With differences and similarities, political spaces incorporated this thematic dimension to their electoral strategies. Considering graphics, print and digital and audiovisuals from each political space, the article analyzes the declarative and thematic ways in which the discussion of urban security as a
\end{abstract}


center of contemporary local politics unfolds. Aires city.

Key words: Insecurity, politics, communication, campaigns. Buenos

\section{Rumo a um mapa das intervenções eleitorais locais em matéria de segurança urbana (Cidade de Buenos Aires, 2007)}

Resumo: Durante os anos noventa de modo similar do que aconteceu na América Latina, as agendas políticas, mediáticas e sociais da cidade de Buenos Aires passaram a ser afetadas com os problemas de segurança urbana. Essas tensões cobraram corpo em 2007, durante as eleições para chefe de governo da cidade, quando uma nova era abriu-se na política local e nacional através da primeira vitória da Proposta Republicana (PRO). Durante esta eleição, a violência urbana e as formas de intervenção contra este problema foram centrais. Com diferenças e semelhanças, os espaços políticos incorporaram esta dimensão temática e suas estratégias eleitorais. A partir de um levantamento de dispositivos gráficos, audiovisuais e digitais de cada espaço político, refletimos sobre as formas declarativas e temáticas em que se desenrola a discussão da segurança urbana como centro da política local contemporânea. Aires.

Palavras-chave: Insegurança, política, comunicação, campanhas, Buenos

\section{Introducción}

"Los siguientes hechos ocurren entre las 19 y las 19.18 pm. No es seguro que el candidato a jefe de Gobierno de PRO, Mauricio Macri, 'mire mucho la serie 24', como le endilgó Jorge Telerman en el debate de la semana pasada. Pero el diputado de licencia emula el minuto a minuto de ese programa en una carrera contra reloj: 24 propuestas en 24 horas. Sobre el final de la campaña, lo sigue un ejército de candidatos y jóvenes del partido, vestidos con una remera amarilla a lo McDonald's, con el logo de PRO. El puntapié lo da el estandarte que llevó el empresario durante toda su campaña: la seguridad. Las propuestas ocurren en tiempo real. 19.01. Llega en moto. Se baja. Se saca el casco. Finalmente no es Kiefer Sutherland, el actor de la serie norteamericana. Es Macri. Sonríe (y) la sede de PRO es una explosión de carteles amarillo huevo”.

El periodista Werner Pertot relata en el matutino argentino Página 12 el 31 de mayo de 2007 los pormenores del cierre de una campaña que instalaría un novedoso modo de hacer política electoral en la Ciudad de Buenos Aires. El periodista también marca las huellas que subrayan un problema de agenda que se termina de imponer durante el proceso electoral: la seguridad, un tema que ya venía siendo tematizado por Mauricio Macri desde su primera participación electoral en 2003. ${ }^{1}$ La violencia urbana es un tema que define la agenda política en la elección a Jefe de Gobierno de 2007 
pero que de ninguna forma está exenta de su propia historicidad local y regional.

A mediados de los noventa la seguridad ingresa con fuerza en las agendas periodísticas, políticas y electorales y se convierte en un eje central de difusión de una comunicación política asociada a la violencia urbana en América Latina en general, y Argentina en particular. No sólo esto se debió a la ampliación del problema del delito en la calle (Sozzo, 2011) basado en la profundización de los índices de denuncias sobre crímenes. A su vez, las discusiones políticas alrededor de la seguridad en la Ciudad de Buenos Aires incorporaron un nuevo matiz local al producirse el estatus de territorio autónomo. ${ }^{2}$ ¿Qué hacer con la policía? ¿Cómo gestionar la experiencia ciudadana de inseguridad?, fueron algunos de los interrogantes que ganaron protagonismo a mediados de los noventa en la ciudad capital de Argentina.

Este artículo da cuenta de algunos resultados de una investigación que sus autores vienen desarrollando en los últimos años acerca de la puesta en agenda política del tópico de la seguridad urbana. En estas páginas avanzamos sobre algunas conclusiones sobre los procesos de securitización de la política local, nacional y regional a partir del análisis específico de las modalidades de construcción de la cuestión securitaria durante la elección a Jefe de Gobierno de la Ciudad de Buenos Aires en 2007. Esta campaña es central para comprender la agenda política de la seguridad de un candidato, Mauricio Macri, líder del espacio político de centro derecha Propuesta Republicana (PRO), que comienza a construirse como referencia ineludible de la política local y nacional en Argentina y que luego de dos gestiones y varias elecciones a su favor en la Ciudad logrará alcanzar la presidencia de la Nación en 2015.

Para avanzar sobre el análisis trabajamos con el relevamiento de los materiales de campaña de los candidatos en disputa en la elección local de 2007, especialmente los tres centrales: Mauricio Macri, Daniel Filmus (del Frente para la Victoria -FPV-, candidato del espacio liderado por el entonces presidente de la Nación, Néstor Kirchner). y Jorge Telerman ${ }^{3}$. Para revisar las regularidades y diferencias alrededor de la cuestión criminal se relevó el material de campaña durante el mes anterior a la primera vuelta electoral, sucedida el 3 de junio, y los quince días previos al ballotage, ocurrido el día 24 del mismo mes. Como resultado, se analizaron once spots audiovisuales (tres del Frente Para la Victoria, tres de Más Buenos Aires -+BA- y siete del PRO), las plataformas electorales de las fuerzas políticas en pugna, los blogs y sitios web de los candidatos, la gráfica (21 en total), folletería, el debate televisivo entre los tres principales candidatos y dos discursos de campaña. En relación al uso de Internet y las redes sociales, cabe consignar que en 2007 no constituían un espacio para el cual se diseñaran estrategias particulares. Si bien eran un territorio electoral donde era posible replicar y reproducir las piezas publicitarias destinadas para otros dispositivos, lejos estaba de tratarse de un escenario de competencia y construcción política. Ello sucedió recién en el 2009². 
Asimismo, incorporamos el vínculo entre las agendas mediáticas y las agendas políticas a través de un relevamiento de las principales columnas de opinión política y tapas de todos los días domingos del 2007 de los diarios nacionales Clarín, Página 12 y La Nación; un registro de noticias publicadas en los dos primeros matutinos entre el 20 de mayo y 24 de junio de ese mismo año y el análisis de material de campaña producido por las principales fuerzas. En el conjunto de este material buscamos regularidades alrededor de los siguientes tópicos: fuerzas de seguridad, diagnóstico de la inseguridad, autonomía, causas, víctimas, victimarios y rol del Estado.

Este relevamiento permitió reconstruir el contexto de la contienda, la forma en que ejerció influencia y las maneras en que apareció la cuestión de la seguridad en los discursos y las estrategias de posicionamiento de los candidatos. Todos estos son elementos que posibilitan en las próximas páginas la reflexión sobre la securitización de los procesos electorales latinoamericanos.

\section{La seguridad y sus significados}

El problema del orden, la conservación de la vida y los bienes materiales es una perturbación intrínseca al Estado moderno en general y a las ciudades en particular. La Ciudad de Buenos Aires no se queda al margen de estos procesos históricos. Lila Caimari (2012) recuerda que esta característica se acentúa en la década del treinta, cuando se profundiza la crisis económica, el incremento de la violencia en las calles y los reclamos de la sociedad civil en torno de la violencia. En tanto durante los treinta impera la añoranza al respeto de las normas y la moral dominante, la tecnología, de la mano del automóvil y de las armas, le da un nuevo perfil al crimen de la época. La población ya por ese entonces demanda orden y el campo político define líneas para intervenir frente a la movilización social. La instauración de los edictos contravencionales de 1932 habilita la imposición de una “ley policial” al funcionamiento político. La emergencia vecinal de la época es alimentada por discursos periodísticos que estigmatizan al delincuente y al desocupado. Las crónicas policiales y los relatos escabrosos se encuentran influenciados por la criminología positivista y ocupan un espacio central en las estrategias de la prensa gráfica para atraer lectores. El campo político, todavía, no interviene en clave securitaria, pero sí sobre la necesidad de reimponer un orden y un consenso que parecen perdidos en la ciudad de la crisis de los treinta.

En las últimas décadas, las democracias latinoamericanas están atravesadas por las discusiones acerca de la necesidad de intervenir en clave securitaria. "El tema de la (in)seguridad ciudadana se ha convertido en un asunto de interés público, político y comunicativo” en gran parte de la región, aseguran Germán Rey y Omar Rincón (2008: 35), una conclusión a la que llegan luego de realizar un análisis comparado entre los principales matutinos de Latinoamérica. La tendencia a identificar el par "delincuencia/ seguridad pública” como el principal problema para la ciudadanía no es 
exclusivo de la Argentina sino que se repite en prácticamente todos los países de la región latinoamericana (Lagos y Damert, 2012).

Más fuerzas de seguridad, más sistemas de videovigilancia, ampliación de penas. "Convertir al miedo en un argumento de la política genera rating”, indican Rey y Rincón (2008: 36). Las recetas de política criminal traídas por expertos desde Estados Unidos pernearon los equipos de campaña y de gestión. Waquant ya revisaba esta novedad hacia el final de la década del noventa en el prefacio de Las cárceles de la miseria titulado "Mister Bratton comes to Buenos Aires": "América latina es hoy la tierra de evangelización de los apóstoles del "más Estado” policial y penal, como en las décadas del setenta y del ochenta, bajo las dictaduras de derecha, había sido el terreno predilecto de los partidarios y constructores del "menos Estado" social dirigidos por los economistas monetaristas de América del norte. Así, los Chicago Boys de Milton Friedman son sucedidos por los New York Boys de Rudolph Giuliani y el Manhattan Institute” (2000: 19). El campo mediático y el político se unieron en el pedido de reformas acordes a los novedosos acordes de la violencia urbana.

En estos escenarios una de las principales intervenciones discursivas de los candidatos de la región suele girar alrededor de la urgencia de la modificación de la legislación penal. Especialmente ello sucede frente a hechos con fuerte repercusión mediática cometidos por adolescentes que suelen funcionar como disparadores de debates complejos y vectores de presión sobre las representaciones políticas. Los reclamos más significativos suelen ser la baja de la de edad de imputabilidad y la mayor ocupación policial de ciertos territorios. Antes y después de la contienda de 2007 fueron resonantes mediáticamente numerosos delitos en los que estuvieron involucrados jóvenes.

Las fuerzas políticas en época electoral avanzan sobre propuestas capaces de imprimir horizontes de certidumbre en territorios diagnosticados como violentos. Las elecciones de países como Brasil, México, Argentina y Chile, entre otros de la región pasaron en las últimas décadas a estar atravesados por la cuestión criminal. Según el investigador norteamericano Paul Chevigny (2003) hay una tendencia política a acudir al miedo y al crimen como issue en las campañas electorales que se verifica tanto en países del cono sur como en Estados Unidos. También sobre esta hipótesis trabaja Jonathan Simon (2012) al sostener que la recurrencia a las víctimas en las campañas presidenciales norteamericanas comienza a ser recurrente desde los sesenta. Pese a las claves diferenciales y qué tan subrayado esté el tópico en una elección u otra, en una fuerza política u otra, la cuestión securitaria se cuela desde fines de la década del noventa en gran parte de las campañas electorales locales y nacionales de la región latinoamericana.

La elección porteña de 2007 no escapa al marco regional de securitización de las campañas políticas. Tal es así que ningún espacio quedó fuera de la necesidad impuesta por la agenda de imprimir un sello propio sobre cómo entender la violencia urbana. El tópico de la seguridad 
fue un eje instalado en la agenda electoral y las recurrencias entre los candidatos fue notoria en esta elección. No obstante ello, el modo de significar la seguridad varió de una fuerza a otra. ¿Cómo plantean los candidatos desde sus campañas que se debe intervenir frente a la violencia urbana? El cómo vivir seguros en una gran urbe se determina en las herramientas que proponen las fuerzas políticas. Las diferencias entre fuerzas ya no necesariamente se despliega por las causas de la violencia, porque como plantea Mauricio Macri, quien llegaría a ser jefe de Gobierno por primera vez en esta elección y presidente de la Nación: "Los pobres también sufren la inseguridad”. El arco político mayoritario asume que o bien como causa de problemáticas sociales, o bien como resultado de elementos estructurales del capitalismo o por falta de incentivos, la inseguridad es un problema de todos y la seguridad es un derecho de los habitantes de la ciudad. Un diagnóstico que parece común pero que despliega múltiples miradas y modos de intervenir frente a la violencia.

De allí a que sea necesario entender las diferencias respecto de la definición de seguridad en una y otra fuerza. Para ello se requiere revisar las formas de intervención que se postulan frente a un eje instalado definitivamente como problemático en las campañas latinoamericanas durante los inicios del siglo veintiuno. En la competencia electoral, las intervenciones discursivas vinculadas con la seguridad están determinadas por lo imperioso de encontrar medidas que sean visualizadas como efectivas. "Las medidas con las cuales se identifican los funcionarios electos deben ser penológicamente creíbles”, recalca el criminólogo David Garland. Pero ante todo "deben gozar de credibilidad política y apoyo popular. En la selección de respuestas políticas resultan más atractivas aquellas que pueden ser más fácilmente representadas como decididas, inteligentes y efectivas o simbólicas” (Garland, 2012: 191). Es un problema de apariencia política y de una retórica capaz de insinuarse posible en clave de efectividad práctica.

En este sentido, en este artículo problematizamos las propuestas de los candidatos a la elección de Jefe de Gobierno de la Ciudad de Buenos Aires en cuatro tipos de intervención discursiva dominante acerca de la cuestión securitaria: territoriales, despliegue policial, el camino cientificista, la tensión cambio social-cambio estructural. Si bien los candidatos optan por una u otra estrategia, no por ello la materia significante deja de intercambiarse en los discursos de distintos espacios políticos. Es decir, cada una de estas definiciones discursivas no pertenecen a uno u otro candidato de manera pura, sino que en algunos casos llegan a imbricarse y podría ser un enunciador de un espacio político tanto como otro el que la postule.

\section{a. Intervenciones territoriales}

En campaña, algunos candidatos suelen describir las ciudades como desordenadas, caóticas y peligrosas. El espacio urbano es históricamente un foco privilegiado de los debates sobre la cuestión securitaria. Ello es así debido a que en la hendíasis espacio-poder, en los contactos, interacciones 
y procesos diversos que atraviesan el ámbito urbano, surgen también los conflictos que responden al cruce de actores con realidades, lógicas e intereses heterogéneos en el entramado social. "No existen ideas políticas sin un espacio al cual sean referibles, ni espacios o principios espaciales a los que no correspondan ideas políticas", formula Carl Schmitt (citado por Cavalletti, 2010: 7). El espacio urbano y los discursos de campaña que giran en torno a la seguridad poseen un vínculo estrecho.

En 2007 en la Ciudad de Buenos Aires son numerosas las intervenciones discursivas que asocian el nivel barrial como lugares que pueden transformarse potencialmente en riesgosos. De allí a que una serie de definiciones electorales proponen la necesidad de diagnosticar y modificar el espacio local. Estas retóricas se centran principalmente en las denominadas estrategias de prevención situacional-ambiental, orientadas a controlar aquellas circunstancias temporales y espaciales de producción de las infracciones. Son intervenciones que apuntan a disuadir las pequeñas incivilidades y los delitos simples, principalmente a través del lazo de la vecindad con los agentes de seguridad. Pero también lo hacen en la denominada prevención comunitaria, estrategia que imagina un vecindario activo en la propuesta y la implementación de las políticas de seguridad.

En las retóricas del territorio, las intervenciones situacionales refuerzan la clave de la eficacia y alejan la definición acerca de la práctica de un posible enunciado que pueda ser entendido por los vecinos como "político”. Juan Bautista Alberdi recalcaba en el siglo dieciocho la importancia de configurar espacios locales como espacios administrativos en los cuales los vecinos pudieran ser capaces de llevar adelante sus asuntos privados. "Ocupado el vecindario en los intereses de su patria social (...) ocupados en la mejora de sus caminos, de la instrucción de sus hijos, del lujo y la elegancia de sus ciudades (...) el vecindario se aleja poco a poco de las estériles agitaciones de la vida política" (Alberdi, 1917, citado por Ternavasio, 1991: 29). En elecciones, el político vuelve a recordar la importancia de los pequeños cambios sobre el territorio para afrontar los grandes problemas urbanos.

Pero para definir esas pequeñas transformaciones cotidianas es preciso mapear el peligro de la urbe para luego afilar el ojo interventor de lo local. En esta línea la propuesta de armar mapas del delito es utilizada por varias fuerzas del arco político. Desde el candidato que era jefe de Gobierno, Jorge Telerman, hasta fuerzas políticamente alejadas de sus propuestas, como lo son las de centro izquierda o izquierda. Este es el caso del espacio referenciado en Claudio Lozano ${ }^{5}$ quien llama a través de los medios de comunicación a la "la conformación de un mapa del delito que identifique áreas conflictivas para impulsar políticas de prevención” (Página 12, 21/ 05/2007). ${ }^{6}$ El mapeo es una estrategia que permite desarrollos híbridos, sociales y situacional ambientales. De allí a que de derecha a izquierda, los candidatos puedan adherir sin dificultades auna herramienta situacional que en principio pareciera poseer mayor afinidad con los programas del racionalismo económico o neoconservador (O’ Malley, 2006). 
El candidato del Frente para la Victoria, Daniel Filmus, también se ubica en los márgenes internos de la estrategia. El problema de la seguridad, asegura, está asociado con "la marginación, con la exclusión, con la existencia de lugares de difícil acceso para las fuerzas de seguridad”. El eje social se entremezcla con el situacional para quedar imbuidos. Para avanzar sobre esos espacios complejos hay que intervenir en clave territorial. En palabras de Filmus: "Hay mejoras urbanas que contribuyen, como la mayor iluminación, la poda de árboles y la organización de redes comunitarias de seguridad”. Las técnicas basadas en el riesgo pueden sin inconvenientes vincularse con discursos políticos sociales, de allí a que sea difícil asocial linealmente a la prevención situacional del delito con el neoconservadurismo.

El Estado local interviene a través de la participación vecinal para controlar en territorio la gestión de riesgos, más que definir intervenciones de forma directa sobre los ofensores. Con un mapeo del peligro, el Estado brinda herramientas a los vecinos como responsables de sus actos locales. Los vecinos ayudan al Estado a generar los saberes locales del delito o se responsabilizan de hábitos que los puedan sumir en riesgos en zonas que saben violentas.

El desplegar mapas del delito permite, argumentan impulsores como Jorge Telerman, “generar condiciones que hagan más difícil delinquir”. Condiciones que implican "desde mejorar el espacio público a eliminar las malezas en los baldíos”, colocar iluminación con más potencia en los barrios, cuidar lo que sucede en las plazas, reacondicionar los terrenos del ferrocarril. Cada una de estas actividades, asegura el candidato en su Blog, debe realizarse "en colaboración con los vecinos”, los verdaderos conocedores del territorio y sus peligros”.

La naturaleza de este enfoque es responsabilizante del vecino y “emponderante” de sus prácticas. O’ Malley recuerda en este sentido cómo los lenguajes e imágenes asociadas con la promoción del control de la criminalidad al estilo "juntos contra el delito" "representan a los miembros del público como agentes activos que persiguen sus propios intereses en una relación voluntaria y mutuamente beneficiosa con la policía y otras agencias de gobierno” (2006, 96). Apelar al vecino implica una imagen de construcción colectiva de las estrategias de prevención, a la par de la responsabilización posterior de los actos individuales.

\section{b. El despliegue policial}

De la misma manera que viene sucediendo desde que Buenos Aires se convirtió en Ciudad Autónoma, en 2007 se instaló el debate sobre la necesidad de contar con una policía propia. De hecho, este fue el eje central de la agenda política y mediática de la Ciudad. Varias crónicas, opiniones y consultas a especialistas o políticos dieron cuenta del interés sobre esta problemática. Por ejemplo, en una entrevista del diario Clarín el entonces ministro de Gobierno porteño, Diego Gorgal, arrojaba cifras que revelaban un aumento vertiginoso de la seguridad privada. Según sus afirmaciones, 
los guardias privados superaban en número a los agentes de la Policía Federal Argentina-PFA.Esta situación, en opinión del funcionario, indicaba que la contratación de vigiladores se había incrementado de manera proporcional a los delitos y era una prueba contundente que demostraba la real preocupación de los porteños. Además, el dato se convertía en una muestra de la desigualdad social, ya que el recurso de la seguridad privada se concentraba en los barrios más favorecidos, como barrio norte, lo que indicaría la falta de protección en otras zonas de la ciudad.

En tal sentido, sostenía Gorgal: “El mapa muestra por qué el delito afecta más a los que menos ingresos tienen: son los que dependen del servicio público. Es similar a lo que pasa con la salud y la educación: el de mayores recursos tiene prepaga (sistema de salud pago) y un colegio privado, el otro no. La desigual distribución de los guardias también deja en evidencia que la seguridad privada mueve el delito desde las zonas controladas a las que no lo están, aunque no lo hace desaparecer”. Y sentenciaba: "El edificio que tiene un vigilador en la puerta tiene menos posibilidades de sufrir un robo que su vecino que no lo tiene. Pero desde lo general, el delito sólo se corre, la situación general no cambia” (Clarín, 20/5/2007). El diagnóstico de la gestión ineficaz de la inseguridad quedaba impreso en las palabras del periodista firmante de la nota: "Lo ideal sería que la seguridad pública coordinara la disposición de los policías con la de los guardias”. La sobreoferta de seguridad pública y privada no se reflejaba, bajo esta mirada, en la disminución de los índices de delincuencia.

En este marco, el entonces Jefe de Gobierno y candidato por el Frente Más Buenos Aires, Jorge Telerman, definió ubicar la cuestión de la autonomía de la Ciudad y la gestión de las fuerzas policiales en el centro de su agenda de campaña. Fue así que en abril de 2007 convocó para el 24 de junio, fecha del ballotage ${ }^{7}$, a una consulta popular para saber si el pueblo “está de acuerdo o no en que tengamos policía en la Ciudad”. "Es la única manera de que el pueblo muestre lo que pide a gritos, esto es pedirle al pueblo que se exprese”, reflexionaba. Su ministro de seguridad reforzaba esta idea: "Planteamos la creación de una policía propia que se ocupe de verificar, controlar, supervisar y hacer que se cumplan las normas de la ciudad de Buenos Aires. Queremos otorgar todo lo que permita dar convicción a los vecinos de que hay un Estado que defiende sus derechos” (Página 12, 20/05/2007). La interpelación a "los vecinos" se combinaba con la propuesta de poner en marcha una policía local, de cercanías. Una fuerza policial propia “capaz, honesta, transparente que conozca al centímetro lo que pasa en los barrios y que tenga una relación de cercanía y confianza con la gente”, argumentaba el jefe de Gobierno en su Blog.

La disputa discursiva versaba en el proceso de autonomización de la ciudad y de gestión de las fuerzas de seguridad. “Quizás -reflexionaba Telerman en medio de la campaña- porque hacerse cargo de una policía autónoma es un proceso conflictivo, me parece que en la Ciudad se han buscado atajos para no asumir las responsabilidades que implica gozar de una autonomía plena”. Entre 2006 y 2007 se presentaron siete proyectos 
sobre la creación de una policía propia de la Ciudad, entre ellos los de los legisladores Silvia La Ruffa ("Creación de la Policía Comunitaria”), y Diego Santilli (“Creación de la policía Metropolitana”) (Anitua, 2010: 89). La decisión de la cabeza del ejecutivo de la ciudad ("Nuestra seguridad, nuestra policía, nuestra decisión”, afirmaban los afiches durante la campaña) se mediatizaba con la búsqueda de consenso pre electoral a través de una consulta para la fecha misma del ballotage.

Transformado en el tema de debate de la campaña, las otras fuerzas políticas debieron opinar. Los candidatos se pronunciaron de numerosas formas: reportajes, declaraciones en actos, comunicados, debates televisivos, programas de gobierno, spots o afiches. Varios días antes de la primera elección, todos, con diferencias y coincidencias, sentaron posición con respecto a la necesidad de una policía local.

Si bien el FPV no basó su campaña en el diagnóstico de la necesidad de una fuerza policial, Filmus tuvo que intervenir en esta agenda y se manifestó de acuerdo con el traspaso de parte de la Policía Federal. Aunque aclaró: "No imaginamos tener dos policías en la ciudad. Los 17.0000 efectivos de la Federal van a constituir la nueva policía y hay que aprovechar el traspaso para formarla. Porque tenemos 23 provincias que tienen policía propia y no resolvieron el problema de la seguridad. No es una solución automática. Además, hay que compatibilizar el territorio de las comisarías con el de las comunas” (Página 12, 20/05/2007). Los límites de la policía local se revelaban en la boca del candidato del Gobierno Nacional. La tensión política impedía imaginar que la autonomía porteña repercutiera en la incidencia del Estado central en la policialización de la Ciudad.

Con importantes coincidencias, el candidato del PRO, Mauricio Macri, aseguraba que "para tener una ciudad autónoma, como su nombre lo indica, pedimos el traspaso de la policía metropolitana”. En su discurso, a diferencia del de Daniel Filmus, la autonomía ingresaba de la mano de la gestión policial en la ciudad. "La policía debe estar a las órdenes del Poder Ejecutivo local. De esta forma podemos aprovechar los recursos de acuerdo con las necesidades y requerimientos de cada barrio o zona. Queremos una policía adecuadamente equipada, profesional y bien remunerada que se sienta orgullosa de proteger a los vecinos y que los vecinos confíen plenamente en su fuerza”, recalcaba Macri (Página 12, 20/05/2007). Su retórica de la gestión de la seguridad adquiere el tono de la eficiencia.

La izquierda también incluye el tópico de la seguridad en las agendas electorales de la región. Difícilmente puedan quedar fuera de uno de los tópicos que se establecen como principal preocupación de los latinoamericanos. En general, si bien es una agenda agenda en términos de propuestas, en las últimas décadas están ingresando en su discusión en tanto que buscan diferenciarse de los discursos que proponen una solución punitiva a partir de la definición de la necesidad de promover la inclusión social de los excluidos antes que soluciones penales del tipo exclusión de las fronteras urbanas de los sujetos peligrosos o baja de edad de imputabilidad. 
La elección de 2007 es muy ilustrativa ya que es una de las primeras en las cuales las fuerzas de izquierda deben intervenir en clave securitaria en la Ciudad de Buenos Aires. El resultado es la discusión alrededor de una agenda en principio ajena pero sobre la que deben mostrar definiciones. El traspaso de la policía o la generación de una fuerza propia emerge como tópico preponderante para estas fuerzas. Sobre una línea similar a la de la fuerza de centro derecha presidida por Macri, Claudio Lozano interviene y sentencia: "La jurisdicción sobre el delito debe estar en manos de la Ciudad. Sobre el traspaso y la reforma policial, proponemos un proceso de transición donde se constituya un fondo fiduciario en el que la Nación deposite el dinero que hoy sostiene a la Superintendencia de Seguridad Metropolitana y sobre el cual la Ciudad realice aportes adicionales”. La gestión local de la seguridad debía ser para esta fuerza, absolutamente un poder de la cabeza de gobierno. De allí a que impulsaran una política de desarme de la población civil, garantizando el "monopolio estatal de la violencia; regulación y coordinación pública de la seguridad privada” (Página 12, 21/05/2007).

El MST se posiciona en una vereda opuesta. Patricia Walsh, candidata del Movimiento Socialista de los Trabajadores, rechazó “de plano el traspaso a la Ciudad de la vieja policía, históricamente asignada a reprimir la protesta social y cuya institucionalidad sigue fuertemente cuestionada por sectores importantes de la población y organismos de derechos humanos”. El Partido Obrero (PO) también rechazó la transferencia de la Policía Federal. Estas fuerzas acentúan la idea un "doble discurso que termina en la represión”, según indica en un folleto el Partido de los Trabajadores Socialistas (PTS).

Lo interesante en ambos casos es que el modo en que los espacios de izquierda entienden la dimensión de la seguridad no necesariamente choca con otros referentes políticos de la ciudad. "Seguridad sí, represión no”, definía en su plataforma el MST. La seguridad para ellos era un problema entendido en clave de violencia urbana sobre la cual un cuerpo especial debía intervenir. Para el PO debían disolverse "los aparatos represivos” y crearse "una fuerza de protección de los derechos ciudadanos reclutada por las organizaciones de derechos humanos y populares”. El MST, por su parte, llamaba desde su plataforma a poner en marcha "un Cuerpo de Seguridad Comunitaria, totalmente nuevo y distinto, con jefe civil, concurso público para selección de personal, mecanismos democráticos de elección y revocación de los comisarios, y bajo control de comisiones de vecinos y organismos de derechos humanos". Si bien el tópico securitario no representa una discusión histórica de estas fuerzas, su inclusión en la agenda electoral muestra las diferencias de contrato con el electorado y los modos de intervención diferenciales de acuerdo a su posicionamiento ideológico.

\section{c. Intervenciones cientificistas}

La incorporación del saber científico-técnico para la comprensión y posterior sanción de las ilegalidades tiene su origen a finales del siglo XIX 
con la consolidación como disciplina de la criminología positivista. El consenso de neutralidad y verdad que rodea al quehacer profesional de médicos, psiquiatras y biólogos es trasladado al estudio de las penalidades.

El marco propicio para su desarrollo efectivo fue el de una sociedad que vincula la categoría de progreso o avance social con la invención o innovación en el perfeccionamiento de objetos que agilicen o alivianen las labores y placeres cotidianos. Otros aspectos de igual importancia que gravitaron fue la aparición de los medios de comunicación masivos que incluyeron sus núcleos conceptuales a las crónicas policiales y la alteración del orden establecido que provocó el proceso industrializador, con el consecuente desplazamiento del campo hacia las ciudades de una enorme masa de personas, sin la infraestructura necesaria para albergarlas.

Frente a tal estado de situación, los criminólogos surgidos de esta corriente de pensamiento rechazan la posibilidad de que el delito fuera una mera conducta humana y postulan su raíz genética. Sus análisis se encuentran orientados por los conocimientos de sus campos, como la anatomía, la fisiología y la psiquiatría. En ellos, no encuentran diferencia alguna entre los malhechores y los locos o insanos morales. Tanto unos como otros son como son por su naturaleza. Además, pueden ser detectados somáticamente. Y las causas de sus conductas ilícitas tienen que ver con un atraso evolutivo, un atavismo común en los monos o en el hombre prehistórico, pero que hoy generan comportamientos anormales. (Anitua, 2006: 180).Este tipo de caracterizaciones reemplaza a los "delitos y las penas" propias del pensamiento liberal de la ilustración, por otra cabalmente tecnologicista donde las penas deben ajustarse al grado de peligrosidad de cada individuo.

El tiempo y la mayor presencia de otras perspectivas, suavizaron su formato de presentación pero no excluyeron las ideas centrales de los discursos dominantes que atienden la problemática de referencia. De hecho el efectismo de la programática macrista se centra, por ejemplo, alrededor de propuestas similares que buscan directamente interpelar a un vecino que se identifica como asediado. Asimismo, y además de intervenir en la discusión de la gestión policial autónoma, utiliza herramientas que no requieren mezclarse en la disputa política ya que están dotadas de un aura cientificista. Una de las propuestas del PRO diferencial en este sentido es la puesta en marcha de un banco de ADN para violadores. ${ }^{8}$

En sus intervenciones televisivas, Macri plantea el diagnóstico de la inseguridad y la preocupación vecinal y lo acompaña inmediatamente de su propuesta de campaña. “¿Puedo hacer una propuesta?”, pregunta a los otros candidatos durante el debate en $A$ dos voces. Y sigue, sin pausa: "El tema violaciones es tema gravísimo que ha crecido en la Ciudad de manera preocupante. ¿Cómo puede ser que un instrumento como el banco de datos de ADN para saber si en la escena del crimen un pelo, semen o una gota de sangre detectan que ya es un violador... Un delito que en 99 por ciento de los casos se comprueba que es reincidente?”, se pregunta. Se vuelca sobre el orgullo de ser una ciudad capital y agrega: “¿Cómo puede ser que noso- 
tros no podamos tener ese instrumento? Córdoba lo tiene. Córdoba tuvo que esperar encontrar un violador que violó 100 veces para animarse a poner este instrumento. El mundo lo tiene. Son instrumentos inteligentes. ¿A quién le va a molestar tener ADN registrado si se porta bien?”. El sujeto no peligroso no debe temer, los vecinos están a salvo por herramientas que según el candidato están creadas para controlar a quienes los acechan.

El modo de ubicarse alrededor de esta propuesta por parte de Mauricio Macri es interesante. Mientras los candidatos intervienen en el debate durante cinco minutos y discuten alrededor de la seguridad urbana, Macri insiste en la definición de propuestas. En tanto los contrincantes lo critican y contra argumentan sin alcanzar a esbozar intervenciones alternativas. Telerman busca disputar con Macri y lo cruza: “Estás haciendo demagogia. Esto ataca los intereses de cada una de las personas. Es una fantasía. Donde dice lo que piensa le sale la mano dura, le sale Sobisch ${ }^{9}$, le sale el banco de datos genéticos”. El debate se estipula en términos de mano dura y mano blanda, de seguridad vs. derechos humanos.

La estipulación de esta última contradicción, debe comprenderse en la nueva etapa que generó el presidente Néstor Kirchner al incorporar a la agenda del Gobierno Nacional, el histórico reclamo de Memoria, Verdad y Justicia que impulsaron desde mediados de la década de los '70, las víctimas del Terrorismo de Estado a través de sus organizaciones más representativas como las Abuelas y Madres de Plaza de Mayo. Un acontecimiento que marcó un antes y un después en el desarrollo de esta nueva realidad: la orden efectuada por el primer mandatario para que el general Roberto Bendini, jefe del Ejército, retirara del Colegio Militar los cuadros de los dictadores Jorge Rafael Videla y Benito Bignone. La indicación se cumplió en el acto, ya que se realizó mientras ambos recorrían las instalaciones del edificio castrense. Era el año 2004 y este novedoso impulso de los juicios por delitos de lesa humanidad hizo que numerosos integrantes de las Fuerzas Armadas (FF.AA.) y de las Fuerzas de Seguridad (FF.SS.) fueran al banquillo de los acusados. Los condenados se repitieron por cientos y las audiencias prosiguen hasta el día de hoy.

Dicha orientación política hizo que un sector del arco político, catalogado genéricamente del centro hacia la derecha manifestara su posición contraria, debido a que entendieron y entienden que la superación de los sucesos originados por la última dictadura debe inscribirse en iniciativas que propicien la "reconciliación entre los argentinos" y en la preocupación por las carencias del presente, donde la seguridad de las personas y su propiedad se ven amenazadas por el "avance del delito". Afirman, a su vez, que la respuesta frente a este cuadro de situación no puede ser la regulación y control de las FF.SS por cuentas pendientes con el pasado, sino que se las debe renovar tecnológicamente y darles eficiencia y autonomía en su gestión.

$\mathrm{Al}$ interior de este encadenamiento discursivo se inserta el PRO. Por eso, entre otras consideraciones, la apelación a las víctimas se vuelve recurrente en su líder. Cuando el cientificismo de la propuesta del banco de ADN 
ingresa en una discusión política, Macri apela a la materialidad de las víctimas: "Mañana te voy a mandar a las mujeres violadas a ver qué te dicen. Te estás riendo de todas las mujeres que fueron violadas, les estás faltando el respeto viejo”. La lógica que se explicita en su discurso es la del representante que busca interpelar en clave de herramientas cientificistas a las víctimas y a los ciudadanos preocupados. Salvando las distancias espaciotemporales, estas retóricas se asemejan a las que Jonathan Simon (2011) recuerda se hacen explícitas en el escenario norteamericano de la década del sesenta, donde las campañas electorales "se han transformado en una lucha por quién se muestra dispuesto a ir más lejos en su papel de acusador (...) Los mandatarios deben mostrar que se identifican con la experiencia de victimización delictiva y con el deseo de venganza que provoca” (Simon, 2011: 55). El uso retórico de las víctimas es claro en el discurso macrista de clave cientificista.

En la misma disputa entre seguridad y derechos humanos se inscribe esta necesidad de acentuar la interpelación de un ciudadano en tanto víctima. Las víctimas, para la postura securitatia, son aquellas asociadas a la criminalidad urbana. Esta modalidad interpelativa implica ampliar un significado que desde los espacios de derechos humanos se vincula con las víctimas del terrorismo de Estado (y durante la democracia con las víctimas de la violencia institucional). Desde allí que para los discursos menos inclusivos en clave de derechos, las víctimas de los delitos de lesa humanidad dejen de ser "las víctimas legítimas” para que este significante pase a estar restringido a las (legítimas) víctimas de la violencia urbana.

\section{d. Inclusión vs. cambio estructural}

La discusión acerca de la inclusión social como modo de recuperar el orden se despliega en todo el arco político. Como planteamos, O Malley (2006) indica la existencia de tres estrategias de prevención, la situacional ambiental, la comunitaria y una tercera centrada en las políticas de prevención social. ${ }^{10}$ Estas últimas ponen el foco en los riesgos económicos, afectivos y morales que pueden llevar a un sujeto a convertirse en infractor. Los estereotipos mediáticos y sociales del criminal ingresan en la discursividad electoral y repercuten en el campo político.

Este tipo de disgregaciones deviene significativas, ya que constituyen discursos del orden que señalan el enfoque y la orientación que los candidatos o decisores a cargo de la gestión gubernamental le imprimen tanto a su campaña electoral como a las iniciativas que impulsan desde la conducción de las áreas especificas que entienden en la problemática aquí planteada.

La influencia de los medios de comunicación en las elaboraciones mencionadas es destacada. Al respecto, Silvia Guemureman indica que para el caso de la delimitación de lo peligroso los medios realizan, entre otras cuestiones, un tratamiento diferencial según se traten de delitos cometidos por adolescentes de clase media alta o en su defecto de bajos recursos. La 
operación que describe funciona para el primer tipo de sucesos, en el marco de una tensión entre aquellos "que aspiran a describir los hechos objetivamente, y por lo tanto, no pueden prescindir de los adjetivos calificativos que despiertan esos hechos: son crímenes brutales (...) Los autores merecen sanciones y los que, por otro lado, intentan reubicar los episodios en hechos aislados, o en todo caso, inscribiéndolos en modalidades típicas de abuso de poder o abuso de clase, y naturalizando ciertos comportamientos como esperables conforme a la posición y estatus social. En tanto para la segunda categoría, no hay espacio para la reflexión: se instala automáticamente el eje joven-pobre-delincuente-drogadicto” y peligroso” (Gumureman, 2014).

Es tal la gravitación de dichos discursos en la agenda pública que incluso para quienes en principio entienden la política criminal de manera diferencial, lejos del orden y el control, los escenarios de caos existen en los territorios de pobreza como las favelas y el peligro de los países vecinos acecha a la Ciudad de Buenos Aires: "Es imprescindible evitar que Buenos Aires replique las situaciones de drogadicción, violencia y muerte que viven las favelas de Río de Janeiro y San Pablo”, recalcaba durante el proceso de campaña Claudio Lozano, de Buenos Aires para todos. Si bien el diagnóstico de peligro es similar, el modo de intervenir frente a él procura diferenciarse de los espacios políticos conservadores: "Las políticas de reconstrucción del tejido social -en materia de empleo, vivienda, educación y salud- junto con el combate a la corrupción policial y la conformación de una nueva policía, son las claves para una política de seguridad integral”, asegura Lozano (Página 12, 21/05/2007). Las tácticas de prevención social recuerdan la necesidad de apartar a los sujetos vulnerables de situaciones potenciales de criminalidad a través de mejorar sus condiciones sociales de existencia.

Vale destacar que estas tácticas no operan en forma pura, ni son retóricas necesariamente utilizadas sólo por fuerzas políticas de centroizquierda. No tan alejado de esta perspectiva al ser consultado sobre si con el control del aparato de policía bastaba para revertir el reclamo por la inseguridad, Macri señalaba que esta gestión debía acompañarse con "inclusión social”. Por ejemplo, a través de becas para chicos para que finalicen el colegio, o la creación de condiciones para que las empresas den el primer empleo a los jóvenes recién egresados. La urbanización de las villas, asegura el candidato del PRO, "constituye otro punto fundamental en la lucha contra la inseguridad para evitar que los vecinos que viven allí sean rehenes de los delincuentes, especialmente ahora con los narcotraficantes que se han apoderado de esos barrios” (Página 12, 20/05/2007). El tópico de la seguridad suele estar asociado a los tópicos educativos y económicos. En un perfil de elector de fuerzas de centro derecha como el PRO, es fuerte el vínculo discursivo entre educación escasa, pobreza y seguridad (datos propios, 2016).

La discusión sobre el eje social y la seguridad urbana queda definitivamente instalada durante la segunda vuelta como centro de la disputa 
entre el macrismo y el oficialismo nacional. El Frente para la Victoria, siguiendo una clave evasiva en torno de la problemática securitaria, decide incorporar la cuestión criminal casi tangencialmente en trono de mejoras de políticas sociales. Así, en uno de sus afiches postula como ejes de campaña: "Educación, seguridad y soluciones". Ante la necesidad de incluir el tópico en la agenda, las mejoras estructurales por momentos terminan estando cercanas discursivamente a los factores de protección y riesgo.

Las fuerzas de izquierda también identifican en algunos fragmentos de sus materiales electorales el crecimiento de la inseguridad con la exclusión social. Patricia Walsh, del MST, indicaba que “la ‘inseguridad” se explica por razones de fondo, como la creciente desigualdad social, que genera exclusión” La candidata recalca que las décadas de exclusión convirtieron al territorio porteño en "Capital de la desigualdad”, de allí a que postule la necesidad de realizar cambios profundos con el fin de "imaginar que la inseguridad se pueda transformar en su contrario” (Página 12, 21/05/2007). De todas maneras, el acento discursivo parece cambiar al ubicarse en la definición no de la necesidad de inclusión social sino de "cambios estructurales”. Por su parte, el Frente de Izquierda Socialista Revolucionario (PTS) se pregunta: “¿La derecha alguna vez se preocupó por los muertos del pueblo?”. Y ante la negativa retórica afirma: “No hay que votar por ninguno de los tres. Todos aspiran a que la ciudad siga siendo Buenos Aires Cromañón. Con inseguridad, trabajo esclavo e impunidad para los grandes empresarios.” Los candidatos de estos espacios, según el FIS, buscan "seguridad” para las grandes empresas e inversiones extranjeras. "Son candidatos Cromañón” ${ }^{11}$ a los que llaman a no votar.

En el fundamento de estas consideraciones, existe una realidad social con muchas tareas aplazadas y la persistencia de altas tasas de delitos y ello a pesar de que se registró, por aquellos años y luego del derrumbe del 2001, un aumento positivo de las principales variables de la economía. Entre los cambios positivos se destacó la efectividad de ciertas iniciativas de política pública como la regulación del mercado laboral, la creación de nuevos puestos de trabajo, aumento de las jubilaciones, pensiones y otros beneficios como la Asignación Universal por Hijo. Las dificultades educativas, del sistema de salud y habitacionales siguen siendo una deuda pendiente. No obstante, esta reversión de la desigualdad no fue acompañada por una caída similar de los hechos ilícitos, si la medición se compara con lo computado a finales de la década de los noventa (Kessler, 2014). En la primera década del siglo veintiuno hubo una tendencia “a la disminución, al menos hasta el 2008, de los delitos contra la propiedad y hasta 2010 de los homicidios (...)” (2014:329) y quela situación inclusiva y contradictoria a la vez puede obedecer a una multiplicidad de factores (ninguno concluyente o determinante) a saber: "Más trabajo, pero más estigmatización y menos oportunidades en algunos territorios; más consumo, pero más privación relativa en momentos de reactivación, la conformación de mercados de delitos en décadas pasadas, (y) el sesgo altamente centrado en la acción policial para tratar la seguridad”, como la altísima preocupación del delito en la cotidianeidad de las personas, situación que degrada la calidad de 
vida y altera las relaciones interindividuales (Kessler, 2014: 331). En los barrios periféricos es donde se continúan concentrando los delitos, en especial los homicidios. La desigualdad de clase continúa operando como un factor de peso para este tipo de hechos. Esta fue, por ejemplo, la justificación argumentativa empleada por Telerman y su ministro Diego Gorgal, para impulsar la creación de una policía local con el fin planteado de democratizar el acceso a las fuerzas de seguridad.

De igual manera, las vicisitudes en torno de cómo analizar la pobreza y la desigualdad en clave estructural no se resuelven en el contexto de las materialidades discursivas de una elección. Más bien estos procesos son el emergente de las tensiones propias de un modo porteño de entender la intervención sobre los escenarios inseguros tanto en clave de prevención social como en clave de intervención penal sobre los responsables. Así y todo, sea en una u otra dirección, los asuntos aquí considerados fueron planteados por todas las fuerzas políticas que compitieron en la contienda.

\section{Conclusiones}

En la Ciudad de Buenos Aires, el debate en relación a la seguridad y la cuestión criminal no es un emergente propio de la competencia electoral del año 2007. Se enmarca en una discusión de la agenda política, mediática y social del conjunto de la región latinoamericana, un tipo de problematización que por momentos excede las diferencias entre espacios políticos ideológicamente contrarios. A su vez, y en términos particulares, la discusión securitaria tiene antecedentes históricos y más reciente en el tiempo deben aplicarse las vicisitudes que trajo consigo el estatus de territorio autónomo, situación alcanzada a partir de la reforma de la Constitución Nacional de 1994.

Las propuestas en materia de seguridad esgrimidas por los candidatos en la campaña electoral del 2007 combinaron, al igual que en otras ciudades y países, posturas vinculadas al welfarismo penal (como la necesaria reducción de la pobreza y la marginación para la disminución del delito) con las sanciones punitivas (expresadas en el registro de violadores de Mauricio Macri) y la consulta popular para la creación de una policía local de Jorge Telerman.

Por el contrario, estuvieron ausentes las opiniones favorables a la reformulación de los programas de readaptación social, eje central del paradigma correccionalista. Entre otras cosas, ello corroboró la tesis afirmada por David Garland según la cual nos encontramos ante un declive del ideal rehabilitador. Para el autor, dicha situación es producto de la crisis del estado de bienestar iniciada en la década del setenta. Es el primer indicador, señala, "de que el esquema de la modernidad -que se había fortalecido incesantemente a lo largo de un siglo- estaba comenzando a desarticularse" (2012: 40). 
En su reemplazo se instaló el modelo neoliberal, caracterizado por la valorización financiera y el individualismo, hecho que trajo aparejado el desmantelamiento de las políticas públicas relacionadas a la cobertura social. En vinculación a lo penal, estos cambios, vinieron asociados a una reducción de la inversión en proyectos de reinserción y a un reposicionamiento político desde la víctima. Para Garland “a lo largo de las últimas tres décadas ha habido un claro regreso de la víctima al centro de la escena en la política de la justicia penal. En el 'complejo penal-welfare', las víctimas individuales apenas aparecían como miembros del público cuyos reclamos provocaban la acción del Estado”. Hasta entonces, asume el criminólogo, los intereses de las víctimas se subsumían a un interés más general y no se contraponían necesariamente a los intereses del sujeto infractor. “Todo eso ha cambiado ahora”, corrobora Garland. "Los intereses y los sentimientos de las víctimas -las víctimas mismas, las familias de las víctimas, las víctimas potenciales, la 'figura abstracta de la víctima' -se invocan ahora rutinariamente...” (2012: 46).

El discurso de la no agresión del PRO es un claro ejemplo de esta perspectiva asociada a la necesidad de interpelar a los ciudadanos en tanto víctimas, al igual que el nosotros inclusivo de un futuro de consenso para el desarrollo de tareas en virtud del bien común. Las propuestas de prevención situacional, como la de mayor iluminación en las calles, y la de la creación de un cuerpo policial porteño, manifestadas por casi la totalidad de todos los candidatos para protección de los vecinos, se inscriben asimismo dentro de este universo.

El temor al delito y la sensación de inseguridad acompañan también este proceso de transformaciones. Lo hacen como aspecto cultural y del sistema de medios de comunicación masivo. Garland entiende que esta "sensación de un público temeroso y resentido ha tenido fuerte impacto en el estilo y contenido de las políticas públicas en los últimos años. Se ha redramatizado el delito" (2012: 45). En algunas coyunturas electorales se apela a presentar discursivamente al ciudadano como irascible y cansado, con una demanda centrada en el castigo, mientras al infractor se le desdibujan los rasgos de un sujeto desfavorecido y merecedor de la ayuda pública. El enojo colectivo y las exigencias en clave moral atraviesan los reclamos mediáticos y los planteos políticos.

Frente a este estado de situación, las principales fuerzas políticas de la contienda de 2007 en la Ciudad de Buenos Aires desplegaron distintas estrategias a la hora de abordar la cuestión de la seguridad. El PRO, se movió de acuerdo a un eje que fue desde lo meramente descriptivo a lo pragmático y en tal sentido, se posicionó en la forma y los espacios donde los hechos delictivos irrumpen en la vida cotidiana, como ser el barrio o un comercio.

La sinergia operada por los espacios políticos durante el período alrededor del significante autonomía y su vínculo con las políticas de seguridad se visualiza tanto en la elección como en el horizonte que se abre una 
vez definido su resultado. El candidato vencedor no dudó en tomar el guante del discurso securitario y en la necesidad estatal de intervención frente al diagnóstico común. "No nos vamos a hacer los distraídos. Seguiremos reclamando nuestros derechos: el traspaso de la policía con los recursos correspondientes. Pero si el gobierno nacional insiste en incumplir con lo prometió en campaña con su obligación y la constitución nacional no nos vamos a quedar cruzados de brazos”, aseguró Macri en el primer discurso de apertura de sesiones en la Legislatura de la Ciudad Autónoma de Buenos Aires, el $1^{\circ}$ de marzo de $2008 .^{12}$ El camino de la autonomía territorial comenzaba a ser ligado materialmente a la autonomía securitaria. "Tomaremos el difícil y largo camino de conformar nuestra propia fuerza policial”, adelantó Macri. "Llevará más tiempo y esfuerzo, pero de ninguna manera eludiremos la responsabilidad que los vecinos de la ciudad delegaron en nosotros. En los próximos días estaré presentando ante la sociedad nuestro plan de seguridad para la ciudad, Quiero antes estar seguro de agotar todas las instancias de diálogo pero no esperaremos eternamente”. El nuevo jefe de Gobierno dejaba claro los pasos a seguir luego de una elección crucial para la historia de la Ciudad de Buenos Aires. Sobre ese camino transitaría luego las elecciones que respaldaron su gestión en la Ciudad y sobre él llegó a la vez a la presidencia de la Nación a fines de 2015. El 2007 es, entonces, el comienzo de una nueva historia política en Argentina, un camino que corre en paralelo a la necesidad de intervención en clave penal de la conflictividad social. 


\section{Notas}

${ }^{1}$ Macri crea el Frente Compromiso para el Cambio para competir en la elección de 2003, en la que perdió en el ballotage a manos de la reelección de la fórmula Anibal Ibarra-Jorge Telerman. En 2005 se presenta como candidato a Diputado Nacional por una alianza denominada Propuesta Republicana (PRO) y sale primero en el distrito de la Ciudad de Buenos Aires. Este sería el primer triunfo de los sucesivos que tendría el candidato hasta llegar a la presidencia de la Nación en 2015.

${ }^{2}$ El estatus autonómico de la Ciudad de Buenos Aires fue establecido por la reforma de la Constitución Nacional Argentina del año 1994 en su artículo 129. No obstante ello, las especificidades de su organización se lograron con la propia Constitución de la Ciudad Autónoma de Buenos Aires y la Ley Nacional N² 24.588, ambas de 1996. El régimen adoptado fue el republicano con la clásica división de poderes (ejecutivo, legislativo y judicial) y varios organismos de control como la Auditoría General y la Defensoría del Pueblo.

${ }^{3}$ Jorge Telerman era vicejefe hasta que asumió formalmente la jefatura de gobierno el 13 de marzo de 2006, luego de la destitución de Aníbal Ibarra por el incendio del boliche porteño Cromañón, ocurrido el 30 de diciembre de 2003 cuyo resultado fue de 194 jóvenes muertos. Sus familiares y la oposición política de la Ciudad responsabilizaron al mandatario por la tragedia y promovieron su juicio político. El proceso fue llevado a cabo por la Legislatura porteña, quién se expidió definitivamente el 7 de marzo del 2006 fallando en contra de su continuidad en el cargo.

${ }^{4}$ En 2009 la explosión de las redes sociales para uso electoral se produce con motivo de varios factores, donde se destacan uno de índole internacional y otros dos pertenecientes a la realidad argentina. Según cuenta Natalia Zuazo, el primero tuvo que ver con la eficacia que tuvo la campaña de Barack Obama para la presidencia de los EE.UU, que logró movilizar a unos 13 millones de militantes en Facebook y recaudar una considerable suma de dinero para el uso proselitista. Las otras son la aprobación de la Ley Orgánica de los Partidos Políticos, que estableció las Primarias Abiertas Simultáneas y Obligatorias (PASO) y abrió la posibilidad de distintas etapas y estrategias diferencias para los candidatos; y la sanción de la Ley de Servicios de Comunicación Audiovisual (SCA), que creó límites y reglas para la competencia electoral (2015).

${ }^{5}$ Claudio Lozano es un referente histórico de la Central de Trabajadores Argentinos (CTA). Fue legislador porteño y diputado nacional en varias oportunidades. En 2007 se candidateó a la Jefatura de Gobierno por la agrupación Buenos Aires para Todos, adoptando una postura crítica hacia los otros candidatos.

${ }^{6}$ También impulsado por los candidatos a diputados por la Ciudad de UNA Nación Avanzada (cuya candidatura presidencial se ubicaba en Roberto Lavagna): “En materia de seguridad ciudadana: pondremos toda la fuerza de la ley para castigar a los delincuentes y proteger a nuestra gente”. Para ello, entre otras acciones impulsan "trazar un mapa del delito para identificar modalidades, lugares y horarios donde se deberá concentrar recursos para aumentar la vigilancia del espacio público” (Plataforma UNA).

${ }^{7}$ El sistema electoral porteño indica que el Jefe/a y Vice Jefe/a de Gobierno son elegidos por el voto directo con mayoría absoluta del cincuenta por ciento más uno de los votos. En caso contrario se establece el mecanismo de ballottage o segunda vuelta 
entre las dos fuerzas más votadas, como sucedió en 2007.

${ }^{8}$ El espacio comandado por Lavagna a nivel nacional también seguía una propuesta similar y en su plataforma llama a "Crear un registro de violadores y un régimen de seguimiento y control por parte del Patronato de Liberados”.

${ }^{9}$ Telerman se refiere al gobernador neuquino Jorge Sobisch, socio político de Mauricio Macri hasta que ordenó la represión contra una manifestación de docentes por reivindicaciones salariales. En tal ocasión fue asesinado el maestro Carlos Fuentealba.

${ }^{10}$ También para ampliar este esquema puede revisarse el detallado análisis de Sozzo (2000).

${ }^{11}$ En la madrugada del 30 de diciembre de 2004 se produjo un incendio en el boliche Cromañón, espacio de diversión nocturna ubicado en el barrio de Once. La tragedia ocasionó la muerte de aproximadamente 400 jóvenes. El suceso se produjo en el transcurso de un show del grupo de rock Callejeros hizo estallar el sistema político de la Ciudad. A tal punto que la Legislatura local aprobó, como ya fuera señalado anteriormente, la destitución de Aníbal Ibarra, por aquél entonces en ejercicio de la Jefatura de Gobierno. Años después, el Poder Judicial comprobó las fallas en los sistemas de seguridad y de emergencia del Estado de la Ciudad. También la complicidad, negligencia y corrupción entre funcionarios públicos y empresarios para no cumplir con la normativa vigente en materia de habilitaciones, seguridad, higiene y control de un espacio de tales características. Sin embargo, apenas emergido el hecho, familiares de las víctimas y diversas fuerzas políticas opositoras y de variados componentes ideológicos denunciaron lo sucedido activamente. Desde los inicios, las agrupaciones de izquierda incorporaron el hecho a su programa de acción política.

${ }^{12}$ Discurso del $1^{\circ}$ de marzo de 2008. trascripto en elparlamentario.com. Disponible en: http://www.parlamentario.com/noticia-13287.html Fecha de última visita: 22 de mayo de 2015 
Polis, Revista Latinoamericana, Volumen 15, No 44, 2016

\section{Bibliografía}

Anitua, G. (Director) (2010), La Policía Metropolitana de la Ciudad de Buenos Aires, Ad-Hoc, Buenos Aires.

Caimari, L. (2012), Mientras la ciudad duerme. Pistoleros, policías y periodistas en Buenos Aires, 1920-1945. Siglo Veintiuno, Buenos Aires.

Cavalletti, A (2010), Mitología de la seguridad. La ciudad biopolítica. Adriana Hidalgo editora, Buenos Aires.

Chevigny, Paul (2003), “The populism of fear. Politics of crime in the Americas”. Punishment\&Society. Nol 5 (1).

Garland, David (2012),La cultura del control. Crimen y orden social en la sociedad contemporánea, Gedisa editorial, Barcelona.

Guemureman, Silvia (2014), “Los ‘unos’ y los ‘otro’: Del tratamiento judicial que reciben los adolescentes y jóvenes que cometen delitos”. Revista de la Facultad de Ciencias Sociales, UBA, N85.

Kessler, Gabriel (2014), Controversias sobre la desigualdad. Argentina, 2003-2013, Fondo de Cultura Económica, Buenos Aires.

Lemke, T. (2010), “Los riesgos de la seguridad: liberalismo, biopolítica y miedo” en Lemm Vanesa, (editora) Michel Foucault, neoliberalismo y biopolítica. Universidad Diego Portales, Santiago Chile.

O’Malley, Pat. (2006), Riesgo Neoliberalismo y Justicia Penal. Ad Hoc, Buenos Aires.

Rey, Germán, Rincón, Omar (2008): “Los cuentos mediáticos del miedo”, Revista Urvio. Revista Latinoamericana de Seguridad Ciudadana. N 5 FLACSO Quito.

Sozzo, Máximo (2000),“Seguridad urbana y tácticas de prevención del delito”, en Cuadernos de jurisprudencia y Doctrina Penal N 10. Buenos Aires: Ad Hoc.

Sozzo, Maximo (2011). "Política penal, elites y expertos en la transición a la democracia en Argentina”. En Nova Criminis. Visiones criminológicas de la justicia penal, $\mathrm{N}^{\circ} 2$. Pp. 147-193.

Sozzo, Máximo (2003), “¿Contando el delito? Análisis crítico y comparativo de las encuestas de victimización en la Argentina”, en Cartapacio de Derecho, Vol 5.

Ternavasio, (1991), “Municipio y política”, un vínculo conflictivo”, Tesis de maestría, Flacso Buenos Aires. Disponible en: http:// 
biblioteca.clacso.edu.ar/

Wacquant, Loic (2000), Las cárceles de la miseria, Manantial, Buenos Aires.

Zuazo, Natalia (2015), “Internet, nuevo terreno de campaña”, en Le Monde Diplomatique, $\mathrm{N}^{\circ} 187$.

Recibido: 17.07 .16

Aceptado: 30.07.16 